\title{
Encoding Finesse Using Dynamic Movement Primitives and Low-Cost Sensors
}

\author{
Ai-Ping $\mathrm{Hu}^{*}$ Colin Usher Michael Matthews
}

\begin{abstract}
Dynamic movement primitives use the output of differential equations to form a description of a demonstrated motion task. Nonlinear forcing functions are used as inputs to the differential equations, which permits a varied range of outputs. By parameterizing the forcing function inputs, it is possible to categorize the resulting output motions. In this paper, dynamic movement primitives are used to encode finesse by characterizing the proficiency level of dextrous tasks performed by people. The demonstration data is recorded using low-cost sensors. Experimental results are presented that indicate it is possible to discern the actions of a novice from an experienced practitioner performing a bird re-hang task common to the food processing industry.

Index Terms-dynamic movement primitives, Kinect, motion characterization, robotics
\end{abstract}

\section{INTRODUCTION}

The tradition of apprenticeship is an age-old practice by which expert knowledge and skills are passed from one generation to the next. As robots are beginning to assist in increasingly sophisticated tasks that were once the exclusive domain of human practitioners (surgery, navigating in unstructured environments, complex decision-making, to name just a few), there has been considerable research devoted to the subject of how to teach these new machines old tricks. The present paper draws an example from the food processing industry to study how human "finesse" in performing manual tasks may be encoded and then used to teach robots to perform more like their human counterparts. The particular task considered is bird re-hang, shown in Fig. 1.

The encoding method implemented uses dynamic movement primitives (see [1], [2], [3]) and low-cost sensors (to acquire demonstration data). Dynamic movement primitives use the output of differential equations to describe a demonstrated motion task. Nonlinear forcing functions are used as inputs to the differential equations, which permits a varied range of outputs. By parameterizing the forcing function inputs it is possible to categorize the output motions, e.g., to distinguish between a novice and an expert. Details of the method are developed in the next section, followed by a description of experiment design (utilizing the low-cost sensors), report of results, and conclusions.

\section{METHOD}

Nonlinear differential equations can exhibit a wide variety of behavior [4]. Dynamic movement primitives (DMP) make

Food Processing Technology Division

Aerospace, Transportation \& Advanced Systems Laboratory

Georgia Tech Research Institute, Atlanta, GA 30332, USA

*ai-ping.hu@gtri.gatech.edu

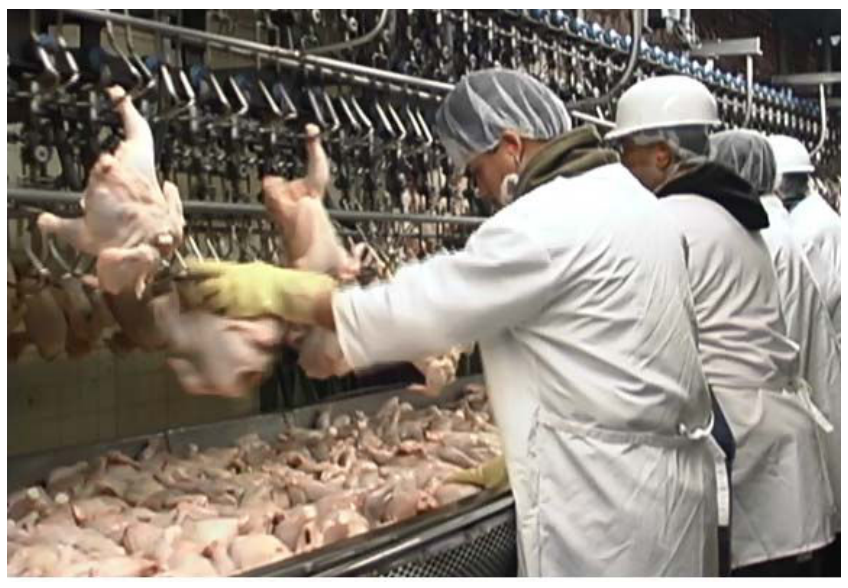

Fig. 1. Bird re-hang inside a poultry processing plant. The operator selects a bird from an unstructured pile and mounts its legs onto a moving shackle.

use of this fact to represent complex motor behaviors, without the need for manual parameter tuning and the danger of instability of the equations [3]. Consider the second-order system

$$
\tau \ddot{y}=\alpha(\beta(g-y)-\tau \dot{y})+f,
$$

where $y(t)$ is designated as the system output as a function of time, $t$, denotes differentiation with respect to time, $g$ is a desired set point, $\tau$ denotes a time constant parameter, $\alpha$ and $\beta$ are constant gains, and $f$ is the nonlinear forcing function. By properly specifying $f$, it is possible to achieve a desired $y$ and $\dot{y}$, which are used as the states defining a desired trajectory. The trajectory may represent, e.g., desired robot joint values or desired Cartesian coordinate values.

Suppose there is a demonstrated trajectory, $y_{\text {demo }}(t)$, acquired from a human practictioner. Upon specifying the forcing function $f$ to be equal to

$$
f_{\text {target }}=\tau \ddot{y}_{\text {demo }}-\alpha\left(\beta\left(g-y_{\text {demo }}\right)-\tau \dot{y}_{\text {demo }}\right)
$$

and substituting into equation (1), with properly selected constants, it may be shown that $y$ converges to $y_{\text {demo }}$.

The DMP method specifies $f$ as a weighted sum of basis functions, $\psi_{i}(x)$ :

$$
f(x, g, y(0))=\frac{\sum_{i=1}^{N} \psi_{i} w_{i} x}{\sum_{i=1}^{N} \psi_{i}}(g-y(0)),
$$

and proceeds by updating the weights, $w_{i}$, to achieve an approximation of $f_{\text {target }}$ by $f$. The monotonically decreasing system

$$
\tau \dot{x}=-\gamma x
$$



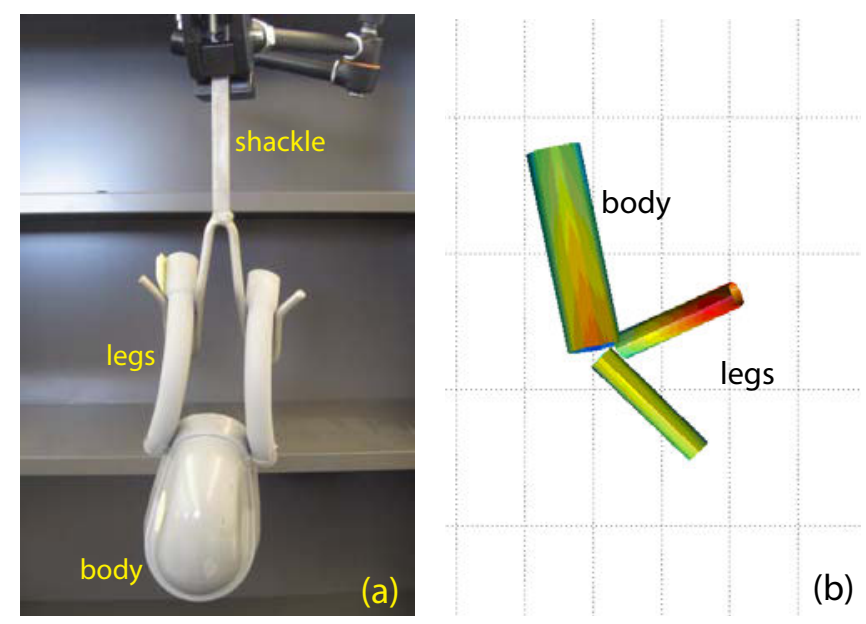

Fig. 2. Bird models: (a) plastic mock-up, shown with shackle and (b) 3-D rigid body MATLAB simulation.

where $\gamma$ is a positive constant, is introduced to allow the variable $x$ to serve as a phase variable, i.e., it is used to indicate which part of the motion we are in and also to synchronize the different joints/coordinates comprising the motion.

The weights, $w_{i}$, are determined by solving

$$
\frac{\sum_{i=1}^{N} \psi_{i} w_{i} x}{\sum_{i=1}^{N} \psi_{i}}(g-y(0))=f_{\text {target }}
$$

at each of the sample points for which demonstration data is available. Generally, the number of sample points exceeds the number of weights, in which case equation (5) is overdetermined (more equations than unknowns) and a leastsquares method may be used to solve for the $w_{i}$.

For ease and consistency, a mock-up bird made up of three plastic pieces are used for the re-hang experiments in this paper. The three bodies are scaled to represent the body and two legs of the bird (see Fig. 2(a)). There is a pin joint between each leg and the body.

Before proceeding to experiments, DMP were formulated based on demonstration data obtained from a numerical model of the bird simulated in MATLAB as three connected rigid bodies (see Fig. 2(b)). The connections were modeled as fairly stiff springs. Fig. 3 shows the results of a typical simulation run for which the three rigid bodies were assigned initial conditions and allowed to interact dynamically. For the example shown, $y_{\text {demo }}$ was taken to be the single " $X$ " Cartesian coordinate of the bird body center of mass over the course of 1 second. The corresponding $\dot{y}_{\text {demo }}$ and $\ddot{y}_{\text {demo }}$ were also readily obtained from the numerical simulation. The time constant and gains in equation (2) were chosen for a stable and relatively fast response. The resulting desired forcing function, $f_{\text {target }}$, and the computed forcing function, $f$ (as a weighted sum of basis functions), are plotted in Fig. 3(a). In this case, $N=15$ and the computed weights are graphed in Fig. 3(b). The computed $f$ is used as the forcing function input to equation (1) and the resulting $y$
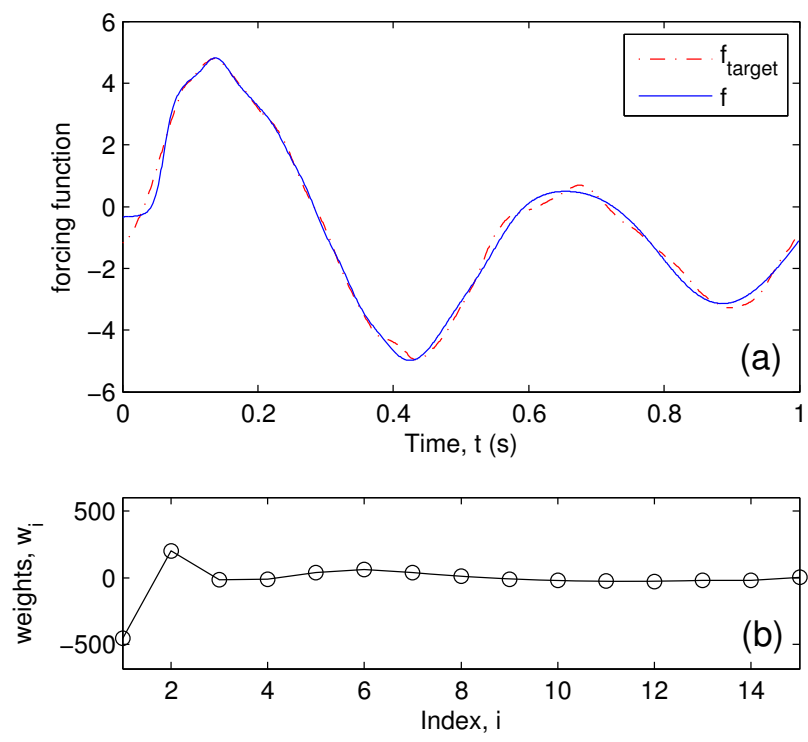

Fig. 3. Sample results from numerical simulation of DMP. Demonstration data obtained from 3-D rigid body simulation output was used to generate $f_{\text {target }}$. The forcing function $f$ is a weighted sum of basis functions.

and $\dot{y}$ are the dynamic movement primitives that may be used as desired trajectories to be taught to a robot.

The weights vary depending on $y_{\text {demo }}$, and may be used to categorize different motions. When the demonstration data comes from human practictioners, the weights may allow distinguishing between different experience levels (beginner versus expert) and characterizing finesse.

\section{EXPERIMENT DESIGN}

Demonstration data for DMP consists of state information, e.g., a position and its time derivatives. In the context of a dexterous task, it is desirable to understand the motions of the performer's hand (or of a manipulated object). This may be accomplished by either measuring joints and applying forward kinematics or by measuring hand motions directly and then applying inverse kinematics to determine the corresponding joint movements.

A main topic of this paper is to investigate the feasibility of using low-cost sensors to obtain the demonstration data for DMP. The low-cost device investigated is shown in Fig. 4. It is composed of an orthogonal coordinate frame having spherical ends and a Shimmer wireless (over Bluetooth) sensing platform affixed to its origin. The coordinate frame is attached to a person's hand and is used in conjunction with a Microsoft Kinect motion sensing gaming device. The Kinect uses an infrared laser projector to determine the depth of points in its field of view. The Kinect has rapidly gained popularity in the robotics community as a low-cost 3-D sensor (e.g., [5], [6], [7]). It is used here to find the Cartesian positions of the three spherical ends of the hand coordinate frame, from which the spatial position and orientation of the hand of the person performing the demonstration task may be computed: the axes comprise unit vectors that may be used 


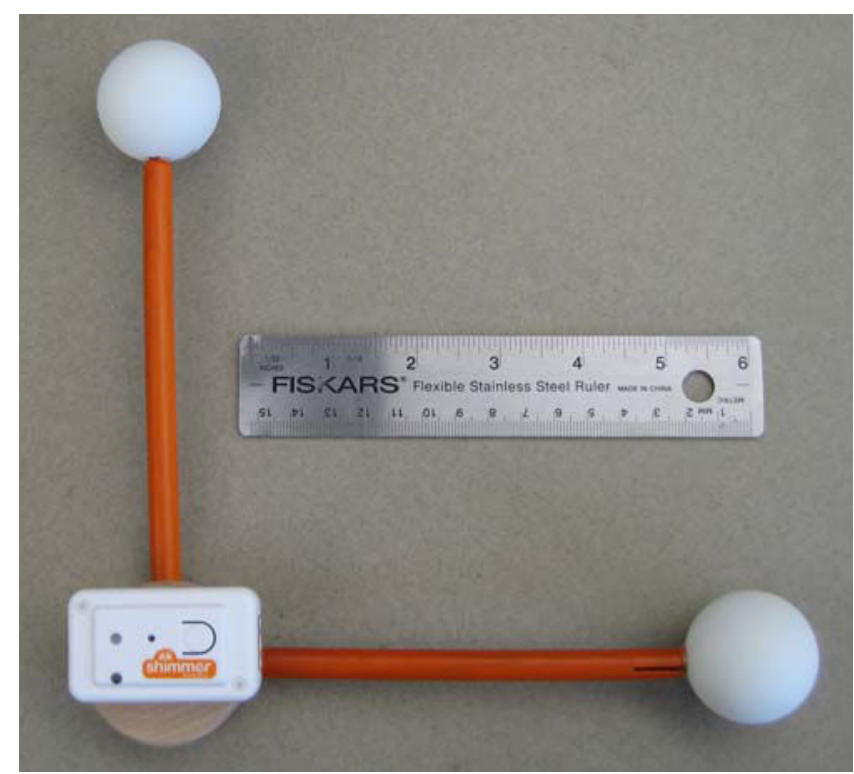

Fig. 4. Coordinate frame and Shimmer module. The Kinect is used to determine the position and orientation of the coordinate frame and the Shimmer measure angular velocities and linear accelerations. Note: for clarity the third orthogonal axis of the coordinate frame is not shown.

to form the columns of the rotation matrix describing the relative orientation of the hand coordinate frame with respect to the Kinect image frame. Fig. 5 shows Kinect depth map images from a sample mock-up bird re-hang demonstration: grasping the bird body and manuevering it to guide the legs into a stationary shackle. The image is colorized to better show the distances to the different objects in the Kinect's field of view. The spherical ends of the hand coordinate frame are readily discernable in the sequence of sample depth images.

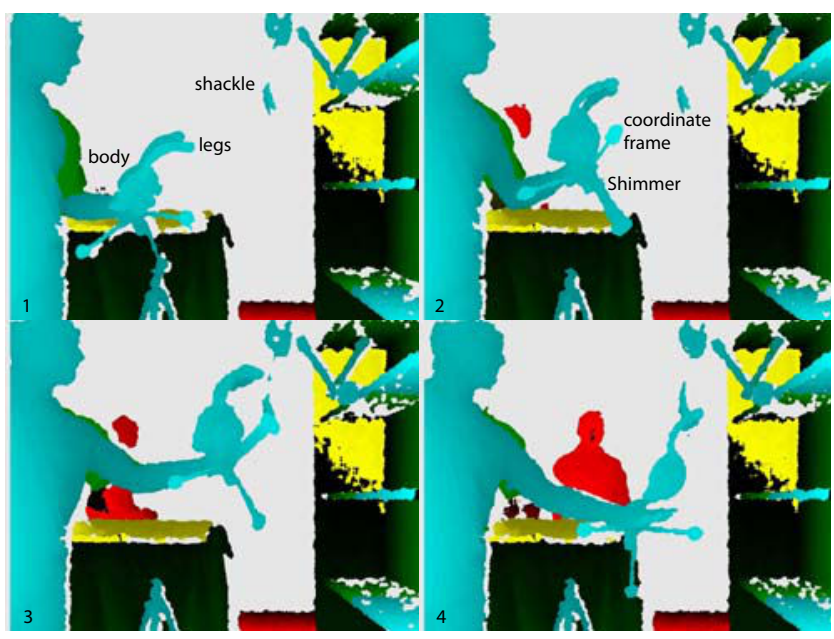

Fig. 5. Laser point cloud depth map images from Kinect. Four frames of a re-hang demonstration are shown, guiding the mock-up bird legs into a stationary shackle.

The Shimmer is a relatively low-cost compact wireless sensing platform used to record and transmit real-time kinematic data. On-board sensors include accelerometers, gyroscopes, and a magnetometer. Shimmer is used to obtain the hand coordinate frame's time rate of change, which is included as part of the demonstration data for DMP. The Shimmer's linear acceleration readings (from the accelerometers) and angular velocity readings (from the gyroscopes) are of interest. The axes of the accelerometers and gyroscopes are nominally parallel with the Shimmer housing, which in turn is aligned with the hand coordinate frame axes (refer to Fig. 4). The linear acceleration and angular velocity readings are therefore defined with respect to the moving hand coordinate frame. A rotation transformation (the same one relating the orientation of the hand coordinate frame to the Kinect image frame) is applied to express these quantities with respect to the Kinect.

During a given demonstration experiment, it is required to also align the timing of the Kinect and Shimmer. This is accomplished by triggering the data acquisition simultaneously. The end result is to obtain $y_{\text {demo }}, \dot{y}_{\text {demo }}$, and $\ddot{y}_{\text {demo }}$ for each of the six outputs comprised of the six Cartesian coordinate variables describing the location and pose of the mock-up bird with respect to the Kinect image frame.

\section{REsults}

Data was collected for multiple re-hang experiments with the mock-up bird, using the experimental method described in section III while executing the general motion depicted in Fig. 5. The stationary Kinect Cartesian image coordinate frame is used and, for discussion below, the " $Y$ " position of the origin of the experimental hand coordinate frame will be defined as the DMP output, $y$. Physically, $Y$ corresponds to the vertical direction (i.e., the direction along gravity) in the Kinect field of view, with positive sense up.

Similar to Fig. 3 for the 3-D rigid body simulation, Fig. 6 shows the DMP forcing function and learned weights based on an example bird re-hang demonstration. The demonstration was recorded after the human practictioner was given time to practice and the task was performed using the person's dominant (right) hand. The demonstration data ( $y$ demo, $\dot{y}_{\text {demo }}$, and $\left.\ddot{y}_{\text {demo }}\right)$ are shown alongside the DMP outputs $y$, $\dot{y}$, and $\ddot{y}$ in Fig. 7. The positional $y_{\text {demo }}$ data is obtained using the Kinect depth map, sampling at $30 \mathrm{~Hz}$. Observe that the $y_{\text {demo }}$ motion returns to approximately the same position, which is in accord with Fig. 5 which shows that the vertical height of the bird after its legs are in the shackle (frame 4) is about the same as the initial height (frame 1). To

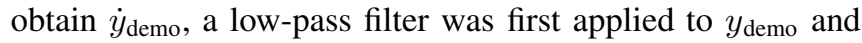
then a numerical derivative of the filtered data was taken. The demonstration acceleration $\ddot{y}_{\text {demo }}$ was obtained from the Shimmer device's accelerometer, properly transformed to the stationary Kinect image frame.

Observe that $\ddot{y}_{\text {demo }}$ contains isolated peaks in acceleration (Fig. 7). This is due to the mock-up bird legs colliding with and rebounding from the metal shackle, which acts as an impulsive force transmitted through the bird body to the task performer's hand. It is not part of the demonstration motion that should be learned by the DMP and it is seen 

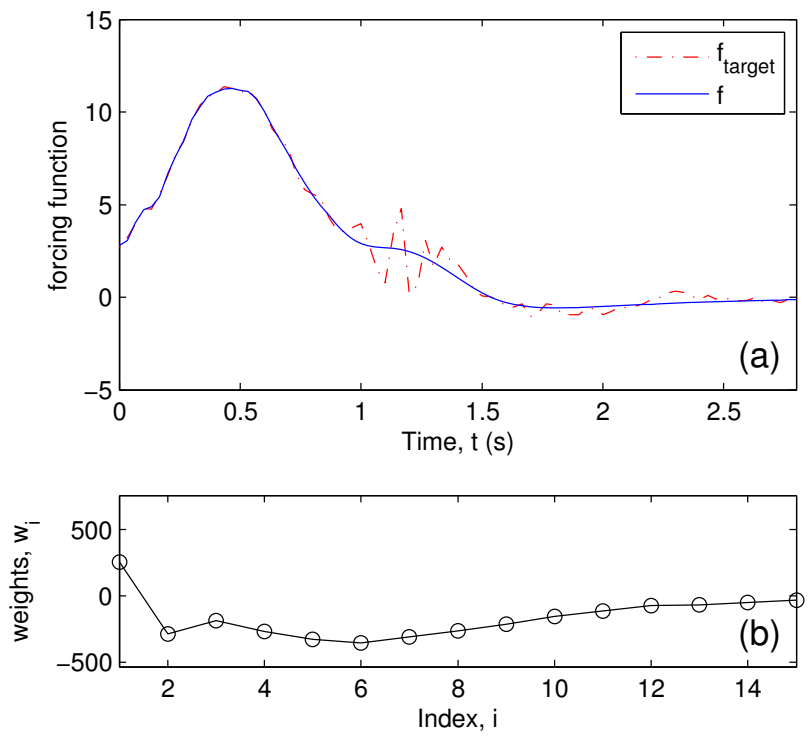

Fig. 6. Example DMP results based on bird re-hang demonstration data from a human practictioner.
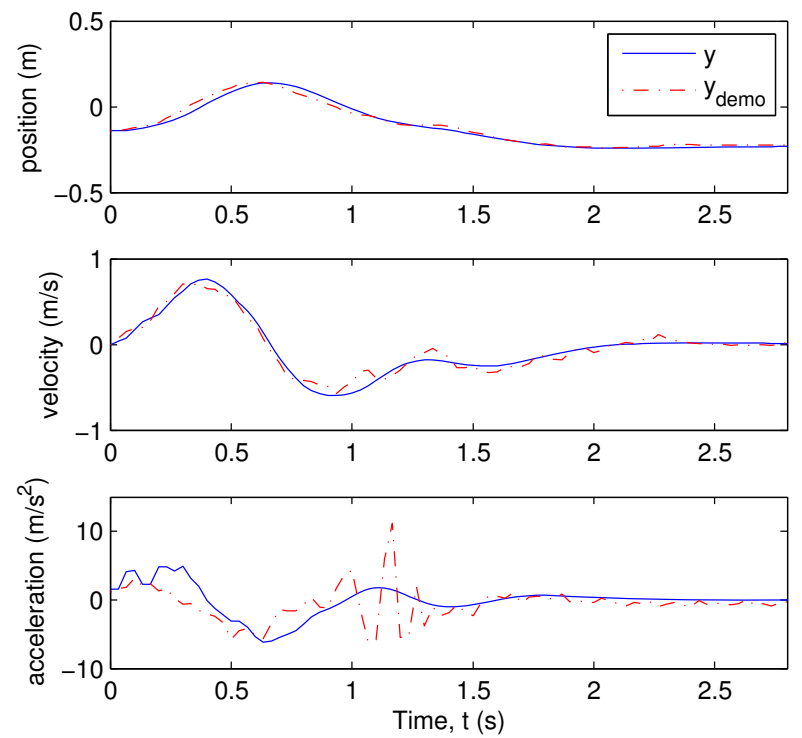

Fig. 7. DMP outputs $y, \dot{y}$, and $\ddot{y}$, shown alongside the corresponding bird re-hang demonstration data.

that $\ddot{y}$ deviates from $\ddot{y}_{\text {demo }}$ in this part of the motion. From Fig. 6(a), observe that the output forcing function $f$ does not approximate the portion of $f_{\text {target }}$ that contains this collision component.

Several experiments were performed with the same task performer executing bird re-hang, yielding very similar results each time. In particular, the learned weights all had a similar shape, magnitude, and trend to those shown in Fig. 6(b). This result is in agreement with published results in [1] and [2], which considered reaching and handwriting demonstration tasks. The novel finding is that similar results could be achieved using relatively low-cost sensors.
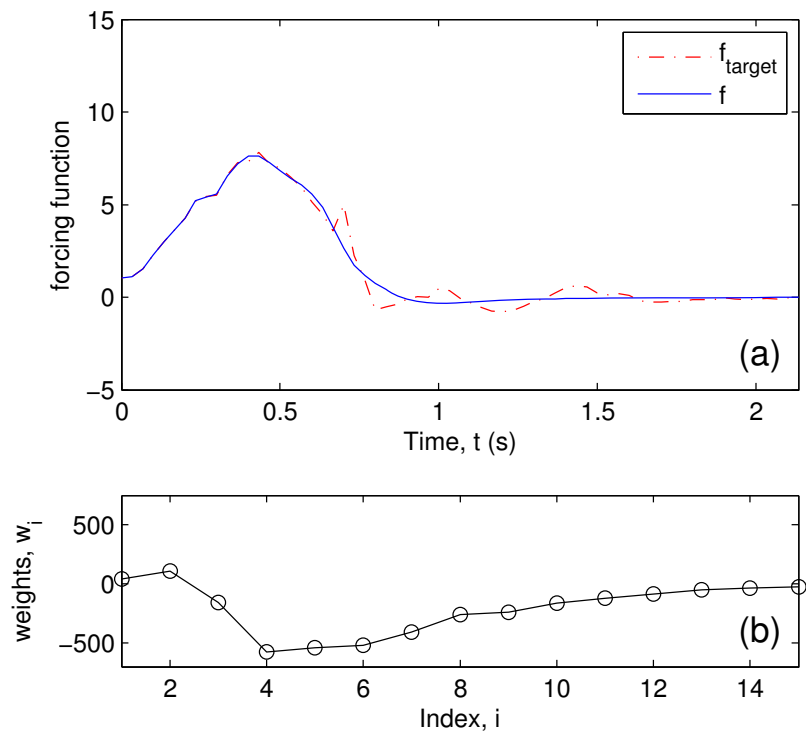

Fig. 8. Example bird re-hang DMP results using non-dominant hand. Results after first trial are shown.
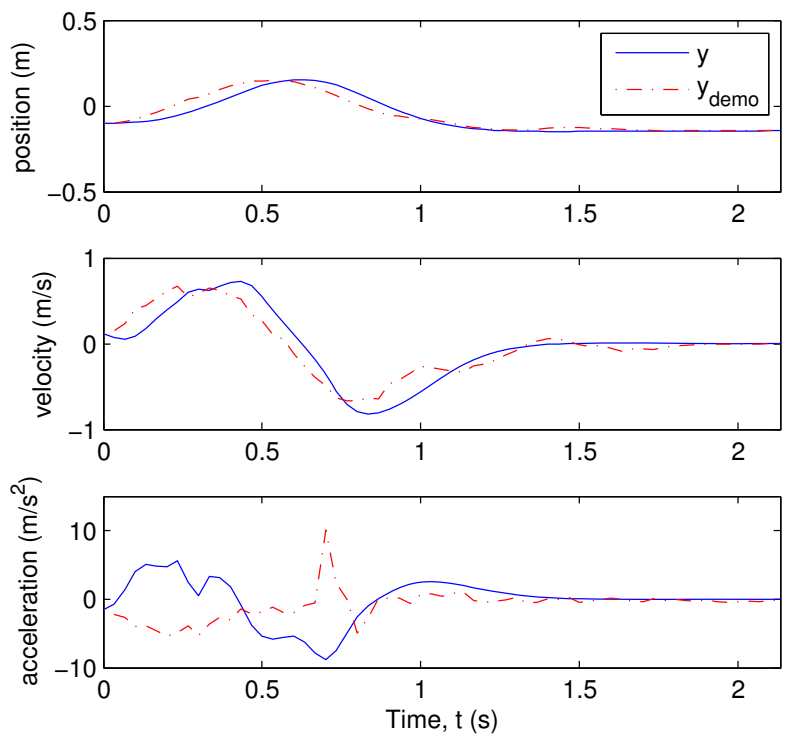

Fig. 9. DMP outputs $y, \dot{y}$, and $\ddot{y}$, shown alongside the corresponding bird re-hang data from a demonstration using non-dominant hand. Results after first trial are shown.

Bird re-hang demonstrations were also performed using the same human practitioner's non-dominant (left) hand. Fig. 8 shows the resulting DMP forcing functions and weights. When compared to the forcing functions and weights corresponding to the dominant hand performing the bird re-hang task in Fig. 6, there is a marked difference. There is also a discernable difference in the non-dominant hand DMP output shown in Fig. 9, when compared to Fig. 7.

This difference in results between the dominant hand and the initial trial with the performer's non-dominant hand is expected, since the non-dominant hand will not be as 

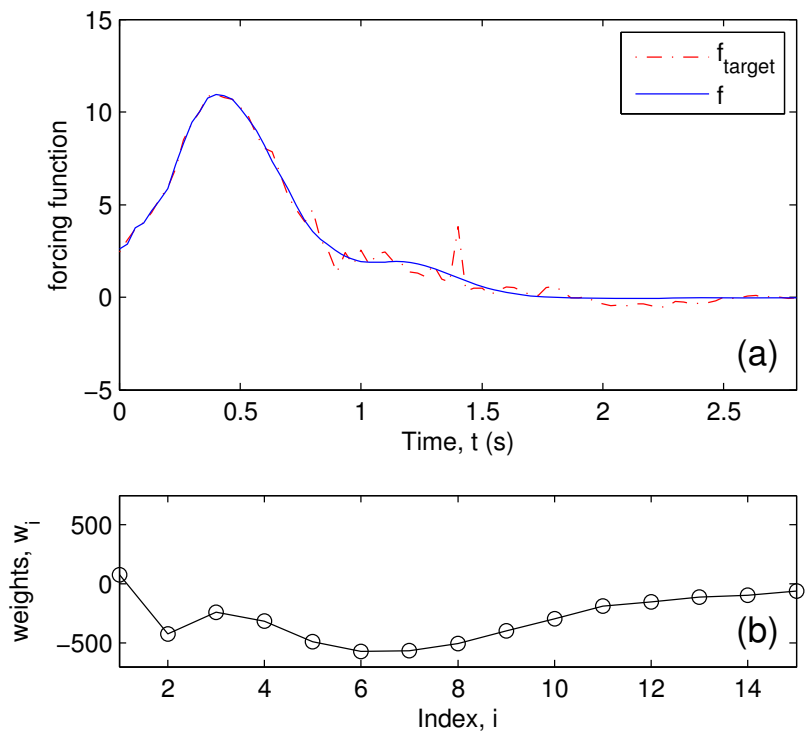

Fig. 10. Example DMP results based on bird re-hang demonstration data from a human practictioner using non-dominant hand, after multiple trials.

proficient in performing the target task. (Recall that the demonstrations with the dominant hand were recorded after the human practitioner was given time to practice the bird re-hang motions.) However, it was found that after being given multiple trials to practice with the non-dominant hand, the human practictioner's re-hang motions approached those of the dominant hand. This is shown by the DMP forcing functions and weights in Fig. 10, which were recorded after several bird re-hang trials had taken place. Observe that the shape, magnitude, and trend of the forcing functions and weights very closely resemble those of the practiced, dominant hand in Fig. 6.

\section{Conclusions}

From the intial results described, a low-cost sensor system consisting of a hand coordinate frame, a Kinect motion sensing gaming device, and a Shimmer wireless sensing platform has been developed that is able to effectively measure hand motions of a human while performing a manual demonstration task. Bird re-hang was chosen as the demonstration task in this paper. It was shown that the sensor system could be used to obtain demonstration data for designing dynamic movement primitives that are capable of discerning motion nuances to distinguish between a proficient practictioner and a novice.

Hand motion during task performance requires six Cartesian coordinates (positions and orientations) to be fully described. The output of a DMP may be designed for each of these coordinates and the resulting sets of weights together comprise a characterization that encodes the finesse associated with the performed demonstration motion. These DMP outputs may then be used as desired trajectories for a robot end-effector, imparting some degree of human finesse to the machine.

\section{ACKNOWLEDGEMENTS}

The authors thank Tyler Vaughn for his assistance in obtaining the experimental demonstration data used in this paper. This work was sponsored by the Agricultural Technology Research Program (ATRP) at the Georgia Tech Research Institute with support from the State of Georgia in cooperation with the Georgia Poultry Federation and its member companies.

\section{REFERENCES}

[1] Ijspeert, A.J., Nakanishi,J., and Schaal, S., "Movement imitation with nonlinear dynamical systems in humanoid robots," Proceedings of the IEEE International Conference on Robotics and Automation, Washington, D.C., USA (2002) 1398-1403.

[2] Ijspeert, A. J., Nakanishi,J., and Schaal, S., "Learning attractor landscapes for learning motor primitives," Advances in Neural Information Processing Systems 15 (2002) 1523-1530.

[3] Schaal, S., Mohajerian, P., and Ijspeert, A., "Dynamics systems vs. optimal control-a unifying view," Progress in Brain Research 165 (2007) 425-445.

[4] Strogatz, S.H., Nonlinear Dynamics and Chaos (Addison-Wesley Publishing Company, Reading, MA, 1994).

[5] Tölgyessy, M. and Hubinský, P., "The Kinect sensor in robotics education," Proceedings of 2nd International Conference on Robotics in Education (2010).

[6] Frati, V. and Prattichizzo, D., "Using Kinect for hand tracking and rendering in wearable haptics," Proceedings of the IEEE World Haptics Conference, Istanbul, Turkey (2011) 317-321.

[7] Khoshelham, S. and Elberink, S. O., "Accuracy and resolution of Kinect depth data for indoor mapping applications," Sensors 12 (2012) 1437-1454. 